\title{
El proceso cognitivo de la toma de decisiones en la enfermedad de Alzheimer
}

\author{
María Rita Malm Morgan¹, Sebastián Urquijo, Fernanda López, Mariela \\ Licitra, Ana Comesaña, Marisa Rodríguez \& Ignacio Brusco
}

\section{Artículo}

Material original autorizado para la publicación en la revista Psicodebate. Facultad de Ciencias Sociales. Universidad de Palermo.

Recibido 22-11-2013 | Aceptado 25-02-2014

\section{Resumen}

El objetivo de este trabajo es describir, desde la neuropsicología, el proceso de Toma de Decisiones (TD) en sujetos que padecen la Enfermedad de Alzheimer (EA). Para ello, se estableció una comparación del desempeño en TD de 40 sujetos con diagnóstico de enfermedad de Alzheimer con el desempeño de un grupo control de 40 sujetos sin diagnóstico de enfermedad neurológica y /o psiquiátrica, pareados por NSEC. El desempeño en la TD se evaluó con la tarea de Game Dice Task (GDT) -Tarea del Juego de los Dados de Brand-. También se aplicaron otras pruebas y tareas de evaluación cognitiva para determinar la relación de la TD con otras Funciones Ejecutivas (FE). Los resultados permitieron establecer que el proceso de TD se deteriora desde los comienzos de la enfermedad, evidenciando que el desempeño de los sujetos con EA es significativamente inferior al de los sujetos sanos, con un porcentaje mayor de respuestas de riesgo y aumentos significativos de los tiempos de respuesta. También se observó deterioro en todas las funciones ejecutivas evaluadas. El trabajo provee evidencia empírica que soporta la idea de que la TD se encuentra estrechamente relacionada a las demás FE.

Palabras Clave: Toma de decisiones (TD) - Enfermedad de Alzheimer (EA) Game Dice Task (GDT) - Neuropsicología.

1 Fundación Humanas - INEAR (Instituto de Neurociencia Cognitiva de Argentina), m.malm. morgan@mac.com 


\section{Abstract}

The purpose of this study is to describe the impairment in the decision making (DM) process in subjects with diagnosis of Alzheimer's disease (AD). The DM performance was assessed on 40 subjects diagnosed with AD and then compared against 40 control subjects with no neurological or psychiatric disease. The DM was evaluated using the Game Dice Task developed by Brand. Subjects were also administered other cognitive assessment tests in order to determine the relationship between the DM with other executive functions. The results show that the DM process seems to be impaired in patients with $\mathrm{AD}$ from the early stages of the disease. The results also show that the DM performance of the subjects with diagnosis of AD seems to be very inferior when compared to that of healthy subjects. DM assessment show that subjects with AD take more risky answers and have a longer reaction time to make the decision than those with no neurological disease. It was also observed that all executives functions seem to be deteriorated as well. This research shows empiric evidence about the relationship between DM and executive functions.

Keywords: Decision Making (DM) - Alzheimer's Disease (AD) - Game Dice Task (GDT) - Neuropsychology. 
En este estudio se investigó la relación entre la Enfermedad de Alzheimer (EA), el proceso de Toma de Decisiones (TD) y el funcionamiento ejecutivo desde el enfoque de la neuropsicología y con instrumentos de evaluación específicos.

La EA es una de las enfermedades neurodegenerativas más frecuentes. Respecto de la incidencia de EA, estudios de cohorte longitudinal sugieren tasas de entre 10 y 15 nuevos casos por cada mil personas-año para todas las demencias y 5-8 para EA (Bermejo-Pareja et al., 2007; Di Carlo et al., 2002), lo que significaría que la mitad de los nuevos casos de demencia cada año son de EA. La edad avanzada es un factor de riesgo para la enfermedad y las tasas de incidencia no son iguales para todas las edades: cada cinco años después de la edad de 65 años, el riesgo de contraer la enfermedad se duplica (Bermejo-Pareja et al., 2007; Di Carlo et al., 2002). También hay diferencias por sexo en las tasas de incidencia. Las mujeres parecen tener un mayor riesgo de desarrollar EA, en particular en la población mayor de 85 años (Di Carlo et al., 2002).

Desde que se evidencian los primeros síntomas de esta enfermedad se generan problemas de gran impacto a nivel social, económico y familiar. Los déficits cognitivos que comienzan a manifestarse generan inconvenientes en la independencia del sujeto. El sujeto no puede estar sólo, lo que genera una situación compleja para la familia. También suelen hacerse presentes otros trastornos conductuales que infringen las normas socialmente aceptadas, los que pueden ir desde la desinhibición, hasta el incumplimiento de la ley. Estos cambios producen alteraciones que son difíciles de afrontar, tanto para quien padece la enfermedad (en los primeros estadios) como para quienes los rodean.

Una de las cuestiones fundamentales a tener en cuenta es qué capacidad tiene el sujeto enfermo de tomar una decisión socialmente correcta, aceptable o adecuada al entorno, cuando sus funciones cognitivas se van deteriorando. En otras palabras, como se ve afectado el proceso de TD durante el curso de la enfermedad.

La vida cotidiana es una sucesión de toma de decisiones, algunas simples, complejas, automáticas y otras necesitan un proceso de evaluación un tanto más exhaustivo. Es en este último caso que algunas personas tienen dificultades en el proceso necesario para decidir cuál es la opción más beneficiosa. Este problema se ve reflejado en muchos casos, tanto cuando las funciones cognitivas se encuentran afectadas y cuyo compromiso afecta a la TD, así como también en adultos mayores aun no evidenciándose patología alguna. Esto último se debe a las alteraciones de la estructura neural en la corteza prefrontal (CPF) asociado a la edad (Sinz et al., 2008).

La TD es el proceso mediante el cual se elige una opción luego de haber evaluado las consecuencias de la misma. Para ello, se requiere del conocimiento previo de ciertos hechos y valores involucrados en la situación. El proceso 
de TD ha sido estudiado en pacientes con daño cerebral frontal, en pacientes con enfermedades neurodegenerativas como la Enfermedad de Parkinson o la Enfermedad de Huntington, y en otras enfermedades psiquiátricas, pero son escasos los estudios sobre la TD en sujetos con EA. En el transcurso de estos años, la mayoría de los estudios neuropsicológicos sobre la TD han utilizado básicamente dos paradigmas para investigar su funcionamiento: el Iowa Gambling Task (IGT) (Bechara et al., 1994) y el Cambridge Gamble Task (CGT) (Rogers et al., 1999). En esta investigación se utilizó un paradigma diferente, el Game of Dice Task (GDT) diseñado por Brand y colaboradores (Brand et al., 2005). Desde su diseño, el GDT ha sido ampliamente referenciado en la literatura científica (Bechara et al., 2005; Brand et al., 2005; Brand, Labbudda \& Markowitsch, 2006; Brand et al., 2006; Brand et al., 2007; Brand et al., 2008; Brand et al., 2009; Bonatti et al., 2009).

Esta investigación es llevada a cabo bajo el enfoque de la clínica neuropsicológica. Esta disciplina estudia el comportamiento de las disfunciones cerebrales; ya sean adquiridas por traumatismos encéfalo-craneanos, como consecuencias de accidentes cerebrovasculares, de intoxicaciones, de enfermedades degenerativas, o psiquiátricas, entre otras causas (Lezak, 1995).

Se considera que esta investigación aporta datos importantes para aquellos profesionales de la salud que se dedican a la asistencia de pacientes con EA. En función de los resultados adquiridos permite tener en consideración el proceso cognitivo de la TD tanto para su evaluación como para su tratamiento de estimulación y así ayudar a que la TD no se deteriore tan rápidamente como otras funciones cognitivas.

El objetivo de esta investigación por lo tanto fue el de explorar el proceso de TD en sujetos que padecen la EA, y establecer una comparación del desempeño de la TD con un grupo de sujetos sin diagnóstico de enfermedad neurológica y/o psiquiátrica, pareado por edad y nivel de instrucción. A su vez, se busca determinar si existe una relación directa entre el desempeño en TD y en el resto de las funciones ejecutivas (FE) ya que éstas se deterioran rápidamente en la evolución de la EA.

\section{Enfermedad de Alzheimer}

La EA es una de las enfermedades neurodegenerativas más frecuentes en el adulto mayor, y afecta al $20 \%$ de las personas de edad mayor a 80 años. Se caracteriza por un inicio insidioso que condiciona un declive cognitivo y funcional progresivo de la persona afectada (Bufill et al., 2009; Martín-Carrasco, 2009). Es poco común que la enfermedad sea sintomática antes de los 50 años de edad, excepto si la EA es hereditaria, aunque son pocos los casos. La mayoría de las personas que presentan esta enfermedad padecen lo que se denomina como EA Esporádica, y su curso tiene una evolución promedio aproximada de una década, 
aunque la progresión es variable en cada individuo (Shannon Rogan \& Lippa, 2002). Desde que se evidencian los primeros síntomas de esta enfermedad, se generan problemas importantes a nivel social, económico y familiar. Los déficits cognitivos que comienzan a manifestarse generan inconvenientes en la independencia del sujeto (Formiga et al., 2008). La memoria episódica es el primer síntoma cognitivo objetivable, debido a esta situación el sujeto que padece la enfermedad ya no debería permanecer sólo, lo que genera una situación compleja para su entorno. Otros déficits cognitivos se evidencian a través de la evaluación neuropsicológica, como por ejemplo alteraciones en las FE y alteraciones en la atención; especialmente cuando se requiere procesar múltiples estímulos simultáneos (Sinz et al., 2008). A medida que la enfermedad progresa, suelen hacerse presentes otros trastornos emocionales y/o conductuales que infringen las normas socialmente aceptadas. Todos estos cambios producen alteraciones difíciles de afrontar, tanto para quien padece la enfermedad (en los primeros estadios) como para quienes lo rodean. El sujeto enfermo deja de ser una persona adulta con independencia de acción y pensamiento y se convierte en un "niño" dependiente de quien lo deba cuidar. Algunos investigadores sugieren que la progresión patológica de la EA aparenta recapitular el desarrollo madurativo descripto desde el concepto de "retrogénesis", definiendo así que las últimas regiones cerebrales en madurar en un desarrollo normal son las primeras en atrofiarse en la EA (Choi et al., 2005).

\section{Toma de decisiones}

La TD es el proceso mediante el cual se realiza una elección luego de haber reflexionado sobre las consecuencias de las diferentes opciones, evaluando las recompensas y los castigos contingentes de cada una de ellas (Clark et al., 2003). Tomar una decisión requiere de conocimiento previo y/o de la interpretación de ciertos hechos y valores involucrados en una reflexión lenta, consciente y con esfuerzo sobre las posibles consecuencias (Bechara, 2006).

El proceso de TD ha sido estudiado en pacientes con daño cerebral frontal y con enfermedades neurodegenerativas como demencia fronto-temporal, enfermedad de Parkinson, el juego patológico asociado a la enfermedad de Parkinson, enfermedad de Huntington, alcoholismo, drogadependencia, aneurisma de la arteria comunicante anterior, epilepsia del lóbulo temporal mesial y traumatismos encéfalocraneanos; pero en la literatura científica son escasos los estudios sobre la toma de decisiones en sujetos con enfermedad de Alzheimer (Bonatti et al., 2008; Bonatti et al., 2009; Brand et al., 2004; Brand et al., 2005; Brand et al., 2008; Brand et al., 2009; Delazer et al., 2007; Delazer et al., 2009; Delazer et al., 2010; Euteneuer et al., 2009; Rossi et al., 2010; Sinz et al., 2008). 
Desde el punto de vista neuropsicológico la TD involucra procesos nocognitivos (emoción) y cognitivos (como la atención, la memoria de trabajo y FE). Debido a esto, este trabajo adhiere a la teoría de Brand (2006, 2007, 2009), la cual sugiere que en el proceso de la TD está también involucrada la CPF dorsolateral (CPFDL), y no únicamente la CPF ventromedial (CPFVM) o la CPF orbital (CPFO). Esta localización también sugeriría que habría una relación directa entre la TD y las FE (Manes, 2002).

La perspectiva de este trabajo tiene como interés el estudio del proceso de TD en sujetos que cursan una patología degenerativa como la EA, ya que a partir de esta enfermedad en particular, se puede observar cómo un proceso cognitivo involucra a otro, y cómo cuando uno de ellos se encuentra deteriorado los otros también se ven afectados, demostrándose así la interconectividad de todos estos procesos.

Siguiendo con esta línea se acepta que las FE estarían involucradas en el proceso de TD. Brand propone un modelo que sugiere que habría dos rutas para decidir de manera ventajosa: una es la estrategia cognitiva, y otra la emocional.

La estrategia cognitiva involucraría los componentes cognitivos necesarios para tomar una elección en función de maximizar los resultados y reducir una mayor pérdida. Se asume que la información relacionada con las características de la decisión a tomar deviene de la memoria a largo plazo (como por ejemplo el conocimiento acerca de probabilidades). Esta información sería monitoreada por las FE, que también son necesarias para categorizar las opciones ofrecidas en la situación, y en donde la memoria de trabajo tendría el rol de combinar la información recibida. En función de este proceso se desarrolla la estrategia a aplicar sobre la decisión a tomar.

La segunda ruta, la emocional, consistiría en el procesamiento del feedback de situaciones previas que podrían activar señales a nivel periférico (señales somáticas) (Brand et al., 2008).

Ante la TD, generalmente existe información explícita sobre las consecuencias potenciales y las probabilidades de recompensas o castigos. En estas situaciones las decisiones se realizan en base a los conocimientos que se tienen sobre la situación, asociados a las consecuencias y a las diversas opciones que se evalúan. A este tipo de TD se la denomina: TD bajo riesgo y está asociada a funciones cognitivasracionales, como el control ejecutivo (Brand et at., 2005; Labudda et al., 2008).

\section{Toma de decisiones y enfermedad de Alzheimer}

En la EA, como en otras patologías, se produce una atrofia frontal. Esto implica que no sólo se manifiestan las dificultades de memoria sino que también aparecen fallas en las FE como pérdida de iniciativa, incapacidad de plantear metas, y/o 
dificultad para evaluar alternativas frente a una situación a resolver, situación que complica aún más el proceso de TD. La TD compleja implica recursos atencionales, procesos ejecutivos y memoria. Consecuentemente, los pacientes con EA manifiestan dificultades en dichas tareas (Delazer et al., 2007). Por lo tanto, resulta razonable suponer que en la evolución natural de la EA, y teniendo en cuenta el consecuente deterioro gradual de las FE, el proceso de TD estaría afectado de forma directa. Así entonces, un estudio detallado del proceso de TD en sujetos normales versus sujetos con EA permitiría obtener información relevante para la comprensión de los procesos cognitivos que regulan la toma de decisiones.

Dentro de las funciones cognitivas alteradas en la EA, se sabe que las alteraciones de la memoria episódica son una de las funciones cognitivas frecuentemente reportadas como motivo de consulta inicial; aunque a través de evaluaciones neuropsicológicas el déficit en las FE ya son evidenciables desde los primeros estadios de la misma. Debido a la interrelación de las FE con la TD, es probablemente razonable suponer que esta última también estaría afectada en los estadios iniciales de la EA.

Desde la neuropsicología, la primera autora que acuñó el término funciones ejecutivas, fue la doctora Muriel Deutsch Lezak, quien las define como "[...] las capacidades cognitivas esenciales para llevar a cabo una conducta eficaz, creativa y aceptada socialmente" (Coolidge, Thede, \& Jang, 2004; Duke \& Kaszniak, 2000; Muñoz-Céspedes \& Tirapu-Ustárroz, 2004; Tirapu-Ustárroz et al., 2005).

Cuando se mencionan las FE se hace referencia a procesos de "alto nivel cognitivo" (inhibición, flexibilidad de pensamiento, resolución de problemas, planificación, control de impulsos, formación de conceptos, pensamiento abstracto, creatividad, organización, la ejecución de comportamientos y los pensamientos complejos) que controlan y regulan procesos de "bajo nivel cognitivo" (percepción viso-espacial, atención visual y auditiva, y memoria a corto y a largo plazo) (Álvarez \& Emory, 2006; Barkley, 2001; Perry \& Hodges, 1999; Pineda, 2000; Sastre-Riva, 2006; Semenova, 2005). Las fallas en estos últimos procesos interfieren con el funcionamiento de los procesos de alto nivel cognitivo.

En síntesis, la EA es una de las demencias más frecuentes en la población de adultos mayores. Como se describió anteriormente, la EA no solo afecta al SNC sino también el funcionamiento social y cognitivo. Si bien desde el punto de vista neurocognitivo los primeros síntomas que manifiesta son dificultades en la memoria episódica, existe también un deterioro de las FE. Esto se evidencia en la incapacidad del sujeto para ser independiente, socialmente productivo, se observa lentificación en el procesamiento de la información, dificultad en organizar la información nueva y antigua, existen dificultades en la flexibilidad cognitiva, como así también, fallas en la inhibición y surgen cambios de conducta, como por 
ejemplo: autocontrol pobre, labilidad emocional, afecto aplanado, irritabilidad, impulsividad y rigidez cognitiva, entre otras (Coolidge, Thede y Jang, 2004). Todo este deterioro no sólo afecta el funcionamiento cognitivo general sino también el proceso de TD en particular.

\section{Método}

\section{Muestra}

Se conformó, de forma incidental, dos grupos pareados por edad y nivel de instrucción.

Criterios de inclusión muestra clínica: Sujetos que padecen la enfermedad de Alzheimer entre 60 y 80 años de edad, con un nivel de educación mínimo de 7 años.

Criterios de inclusión muestra control: Sujetos que no padecen la enfermedad de Alzheimer entre 60 y 80 años de edad, con un nivel de educación mínimo de 7 años y que no presentan deterioro cognitivo según el MMSE y el GDS.

Criterios de exclusión: Se excluyen sujetos con enfermedades neurológicas y/o psiquiátricas que no sean enfermedad de Alzheimer.

Muestra clínica: Un grupo compuesto por 40 adultos con edades entre $60 \mathrm{y}$ 80 años, con nivel de educación mínimo de 7 años y diagnóstico de enfermedad de Alzheimer. Las derivaciones fueron realizadas el servicio de neurología de un hospital público de la Ciudad Autónoma de Buenos Aires al servicio de neurociencias del mismo hospital.

Muestra control: Grupo compuesto por 40 adultos sin diagnóstico de enfermedad neurológica o psiquiátrica, con edades entre 60 y 80 años y con un nivel de educación mínimo de 7 años, pareados por NSEC con los sujetos de la muestra clínica, que concurrieron al servicio de neurociencias de un hospital público de la Ciudad Autónoma de Buenos Aires.

\section{Instrumentos}

Para evaluar el desempeño en los procesos de TD, se utilizó el Game of Dice Task o GDT (Tarea del juego de dados) de Brand y colaboradores (2005), en su versión informatizada. Al comienzo de la prueba se le solicitó a los participantes que traten de maximizar un capital inicial imaginario (\$1000). Los participantes debieron adivinar qué número aparecerá en 18 tiros de un dado. Antes de cada tiro debían decidir entre un número en particular (ej.: 3) o una combinación de dos (ej.: 1 y 2 ), de tres (ej.: 1, 2 y 3 ) o de cuatro (ej.: 1, 2, 3 y 4) números. Los participantes ganaban cuando el número elegido (o la combinación de números) aparecía en la 
pantalla; de lo contrario, perdían. Las ganancias y pérdidas de cada elección son asociadas con la probabilidad de su ocurrencia (ganar/perder eligiendo un solo número es $\$ 1000$, ganar/perder eligiendo dos números es $\$ 500$, ganar/perder eligiendo tres números es $\$ 200$ y ganar/perder eligiendo cuatro números es \$100).

Las alternativas de elección y las ganancias/pérdidas asociadas, estaban siempre presentes en la pantalla de la computadora durante la tarea. Si bien la elección de un número sólo era la opción más riesgosa y desventajosa, la combinación de cuatro números era la decisión más conservadora y ventajosa. Antes de comenzar se instruyó explícitamente a los participantes acerca de las reglas, de las ganancias y las pérdidas asociadas con cada elección, así como de la duración total del juego (18 tiros). Sin embargo, no se les informó acerca de cuál es la decisión más ventajosa. Luego de hacer una elección (y la animación de un tiro del dado), la ganancia o la pérdida, el capital residual y la cantidad de tiros remanentes eran presentados visualmente en la pantalla, y fueron explicados. Señales acústicas indicaban ganancia o pérdida.

Los autores clasificaron las elecciones de uno o dos números como riesgosas y desventajosas, mientras que las elecciones de tres o cuatro números las consideraron como seguras y ventajosas (Brand, Labbuda, \& Markowitsch, 2006; Delazer et al., 2007).

Otras pruebas neuropsicológicas fueron también utilizadas, conformando una batería de tests para evaluar otras funciones cognitivas. Estas pruebas fueron: Mini Mental State Examination o MMSE desarrollado por Folstein et al. (1975), el Functional Assessment Stages o FAST (Reisberg et al., 1985/1992/2000), el Global Deterioration Scale o GDS (Reisberg et al., 1982), el A.D.A.S. -Alzheimer's disease Assessment Scales (Escala de Evaluación para la Enfermedad de Alzheimer)- desarrollada por Rosen y colaboradores en 1984/1986 y adaptada en Buenos Aires por Mangone y colaboradores (1997), el DRS-Mattis Dementia Rating Scale (Escala de demencia de Mattis) - es una escala desarrollada por Mattis (1988), la Fluencia verbal, es una prueba desarrollada por Benton (1976/1989) y Spreen y Strauss (1991), el Test del Tazo con sus partes A y B (Trail Making Test-TMT-A, TMT-B-), el Test Auditivo-Verbal de Rey (TAVR) (Rey Auditory Verbal Learning Test), esta prueba fue desarrollada por André Rey en 1964 (Allegri et al., 1999; Lezak, 1995; Burin, Drake, \& Harris, 2007).

Con el objeto de establecer la presencia de deterioro de los sujetos de la muestra, las puntuaciones de los instrumentos fueron recategorizados, utilizando los criterios de los propios instrumentos y de la literatura, dividiendo a los grupos en función de la presencia de indicadores de deterioro.

Con el fin de determinar la confiabilidad de los instrumentos utilizados para medir la presencia de deterioro se realizó una prueba de correlación. Los resultados se presentan en la Tabla 1. 
Malm Morgan, Urquijo, López, Licitra, Comesaña, Rodríguez \& Brusco I Psicodebate, 14(1) I 9-32.

Tabla 1.

Correlaciones (Rho de Spearman) entre las pruebas para medir nivel de deterioro MMSE-FAST-GDS-ADAS-DRS

\begin{tabular}{|c|c|c|c|c|c|c|}
\hline & & MMSE & FAST & GDS & ADAS & DRS \\
\hline \multirow{2}{*}{ MMSE } & $r$ & & $.948^{* *}$ & $.943^{\star *}$ & $1.000^{* \star}$ & $.940^{\star *}$ \\
\hline & $p$ & & .000 & .000 & - & .000 \\
\hline \multirow{2}{*}{ FAST } & $r$ & $.948^{\star \star}$ & & $.957^{\star \star}$ & $.948^{\star \star}$ & $.941^{\star \star}$ \\
\hline & $p$ & .000 & & .000 & .000 & .000 \\
\hline \multirow{2}{*}{ GDS } & $r$ & $.943^{\star \star}$ & $.957^{\star *}$ & & $.943^{\star \star}$ & $.952^{\star *}$ \\
\hline & $p$ & .000 & .000 & & .000 & .000 \\
\hline \multirow{2}{*}{ ADAS } & $r$ & $1.000^{\star \star}$ & $.948^{\star \star}$ & $.943^{\star \star}$ & & $.940^{\star \star}$ \\
\hline & $p$ & - & .000 & .000 & & .000 \\
\hline \multirow{2}{*}{ DRS } & $r$ & $.940^{\star \star}$ & $.941^{\star *}$ & $.952^{\star \star}$ & $.940^{\star \star}$ & \\
\hline & $p$ & .000 & .000 & .000 & .000 & \\
\hline
\end{tabular}

${ }^{\star \star} \mathrm{p}<.01$

Como se puede observar se verificó una asociación muy alta (superior a 0,94 en todo los casos) y estadísticamente significativa entre las pruebas seleccionadas para establecer el nivel de deterioro cognitivo de los participantes. Se observa especialmente entre el MMSE y el ADAS las cuales coinciden dividiendo a los sujetos de la misma manera y son equivalentes, es decir que sabiendo el resultado de una de ellas se podría inferir el desempeño de la otra.

\section{Resultados}

A continuación, en la Tabla 2, se presentan los estadísticos descriptivos de los resultados de los instrumentos administrados a todos los integrantes de la muestra, para determinar el nivel de deterioro cognitivo, discriminados por tipo de prueba y por grupo.

Tabla 2.

Estadísticos descriptivos de medidas de deterioro según grupo.

\begin{tabular}{|c|c|c|c|c|c|c|c|c|}
\hline \multirow[t]{2}{*}{ Prueba } & \multicolumn{4}{|c|}{$\begin{array}{c}\text { Controles } \\
(n=40)\end{array}$} & \multicolumn{4}{|c|}{$\begin{array}{c}\text { Pacientes } \\
(n=40)\end{array}$} \\
\hline & Mínimo & Máximo & $\mathrm{M}$ & $\mathrm{DE}$ & Mínimo & Máximo & $\mathrm{M}$ & $\mathrm{DE}$ \\
\hline MMSE & 27 & 30 & 29.25 & 0.840 & 10 & 24 & 21.75 & 3.264 \\
\hline FAST & 1 & 2 & 1.43 & 0.501 & 3 & 5 & 3.78 & 0.660 \\
\hline GDS & 1 & 1 & 1.00 & 0.000 & 3 & 5 & 3.53 & 0.599 \\
\hline ADAS & 0 & 9 & 4.60 & 3.581 & 10 & 70 & 21.16 & 9.523 \\
\hline DRS & 130 & 144 & 138.45 & 4.188 & 93 & 129 & 115.68 & 11.155 \\
\hline
\end{tabular}


Las Tablas 3 y 4 muestran los resultados del rendimiento de los sujetos en pruebas de evaluación de funcionamiento cognitivo y de Toma de Decisiones (GDT) discriminados por grupo.

Tabla 3.

Estadísticos descriptivos de pruebas de funcionamiento cognitivo discriminados por grupo

\begin{tabular}{|c|c|c|c|c|}
\hline Prueba & Grupo & $M$ & $D E$ & EEM \\
\hline \multirow{2}{*}{ ADAS Cog } & C & 0.60 & 1.008 & 0.159 \\
\hline & $\mathrm{P}$ & 10.15 & 7.608 & 1.203 \\
\hline \multirow{2}{*}{ ADAS Mem } & C & 4.01 & 3.175 & 0.502 \\
\hline & $\mathrm{P}$ & 11.00 & 2.991 & 0.473 \\
\hline \multirow{2}{*}{ DRS } & C & 138.45 & 4.188 & 0.662 \\
\hline & $\mathrm{P}$ & 115.68 & 11.155 & 1.764 \\
\hline \multirow{2}{*}{ FVF } & C & 17.10 & 4.425 & 0.700 \\
\hline & $\mathrm{P}$ & 8.88 & 3.560 & 0.563 \\
\hline \multirow{2}{*}{ FVS } & $\mathrm{C}$ & 21.08 & 5.413 & 0.856 \\
\hline & $\mathrm{P}$ & 9.63 & 3.801 & 0.601 \\
\hline \multirow{2}{*}{ TMT-A } & C & 39.40 & 12.087 & 1.911 \\
\hline & $\mathrm{P}$ & 105.45 & 37.835 & 5.982 \\
\hline \multirow{2}{*}{ TMT-B } & $\mathrm{C}$ & 95.75 & 31.173 & 4.929 \\
\hline & $\mathrm{P}$ & 288.85 & 43.252 & 6.839 \\
\hline \multirow{2}{*}{ TAVR-A1/A5 } & $\mathrm{C}$ & 46.48 & 7.805 & 1.234 \\
\hline & $\mathrm{P}$ & 21.38 & 7.692 & 1.216 \\
\hline \multirow{2}{*}{ TAVR-A1 } & C & 4.95 & 1.880 & 0.297 \\
\hline & $\mathrm{P}$ & 2.43 & 1.781 & 0.282 \\
\hline \multirow{2}{*}{ TAVR-A2 } & $\mathrm{C}$ & 7.68 & 2.018 & 0.319 \\
\hline & $\mathrm{P}$ & 4.00 & 1.519 & 0.240 \\
\hline \multirow{2}{*}{ TAVR-A3 } & $\mathrm{C}$ & 9.93 & 2.018 & 0.319 \\
\hline & $\mathrm{P}$ & 4.48 & 1.853 & 0.293 \\
\hline \multirow{2}{*}{ TAVR-A4 } & $\mathrm{C}$ & 11.53 & 2.253 & 0.356 \\
\hline & $\mathrm{P}$ & 5.20 & 1.964 & 0.311 \\
\hline \multirow{2}{*}{ TAVR-A5 } & $\mathrm{C}$ & 12.40 & 1.945 & 0.308 \\
\hline & $\mathrm{P}$ & 5.40 & 2.384 & 0.377 \\
\hline \multirow{2}{*}{ TAVR-B } & $\mathrm{C}$ & 5.73 & 1.797 & 0.284 \\
\hline & $\mathrm{P}$ & 2.25 & 1.410 & 0.223 \\
\hline \multirow{2}{*}{ TAVR-A6 } & $\mathrm{C}$ & 9.63 & 2.676 & 0.423 \\
\hline & $\mathrm{P}$ & 2.18 & 2.049 & 0.324 \\
\hline \multirow{2}{*}{ TAVR-A7 } & $\mathrm{C}$ & 9.85 & 2.824 & 0.447 \\
\hline & $\mathrm{P}$ & 1.78 & 2.293 & 0.362 \\
\hline \multirow{2}{*}{ TAVR-R } & $\mathrm{C}$ & 14.65 & 0.580 & 0.092 \\
\hline & $P$ & 7.13 & 3.539 & 0.560 \\
\hline
\end{tabular}


A simple vista, es posible observar, de forma sistemática el déficit en el desempeño de los sujetos de la muestra clínica.

\section{Tabla 4.}

Estadísticos descriptivos de pruebas de TD discriminados por grupo.

\begin{tabular}{lccccc}
\hline & Grupo & $\mathrm{N}$ & $\mathrm{M}$ & $\mathrm{DE}$ & EEM \\
\hline \multirow{2}{*}{ GDT-RC $^{*}$} & $\mathrm{C}$ & 40 & 8.65 & 2.833 & 0.448 \\
\cline { 2 - 6 } & $\mathrm{P}$ & 40 & 7.05 & 2.521 & 0.399 \\
\hline \multirow{2}{*}{ GDT-RR $^{*}$} & $\mathrm{C}$ & 40 & 7.15 & 4.441 & 0.702 \\
\cline { 2 - 6 } GDT-RS $^{*}$ & $\mathrm{P}$ & 40 & 11.00 & 5.796 & 0.916 \\
\cline { 2 - 6 } & $\mathrm{C}$ & 40 & 10.85 & 4.441 & 0.702 \\
\hline \multirow{2}{*}{ GDT-Tpo.RS* $^{*}$} & $\mathrm{P}$ & 40 & 7.00 & 5.796 & 0.916 \\
\hline \multirow{2}{*}{ DGT-Tpo.RR } & $\mathrm{C}$ & 40 & 9.60 & 5.222 & 0.826 \\
\cline { 2 - 6 } & $\mathrm{P}$ & 40 & 11.80 & 10.204 & 1.613 \\
\hline \multirow{2}{*}{ GDT-Tpo.RP* $^{*}$} & $\mathrm{P}$ & 40 & 10.45 & 8.524 & 1.348 \\
\cline { 2 - 6 } & $\mathrm{C}$ & 40 & 12.70 & 6.836 & 1.081 \\
\hline
\end{tabular}

${ }^{*}$ GDT-RC: total de respuestas correctas, GDT-RR: total de respuestas de riesgo, GDT-RS: total de respuestas seguras, GDT-Tpo.RS: tiempo de reacción de respuestas seguras, GDT-Tpo. RR: tiempo de reacción de respuestas de riesgo, GDT-Tpo.RP: tiempos de reacción promedio.

A simple vista es posible observar que existen diferencias en el desempeño tanto en las pruebas de funcionamiento cognitivo como en las de TD de los sujetos del grupo control y los sujetos con EA. Con el objeto de determinar la significación de las diferencias en pruebas de funcionamiento cognitivo y de toma de decisiones se realizó una prueba $t$ de diferencias de medias para muestras independientes. Los resultados se presentan en las Tablas 5 y 6. 
Tabla 5.

Resultados de la prueba t de muestras independientes de pruebas de funcionamiento cognitivo en función de la pertenencia al grupo.

\begin{tabular}{|c|c|c|c|c|c|c|}
\hline \multirow{2}{*}{ Prueba } & \multirow{2}{*}{$t$} & \multirow{2}{*}{$p$} & \multirow{2}{*}{$D M$} & \multirow{2}{*}{ EEDM } & \multicolumn{2}{|c|}{$95 \%$ IC para la diferencia } \\
\hline & & & & & Superior & Inferior \\
\hline ADAS Cog & -7.871 & .000 & -9.550 & 1.213 & -11.966 & -7.134 \\
\hline ADAS Mem & -10.142 & .000 & -6.995 & 0.690 & -8.368 & -5.622 \\
\hline DRS & 12.089 & .000 & 22.775 & 1.884 & 19.024 & 26.526 \\
\hline FVF & 9.159 & .000 & 8.225 & 0.898 & 6.437 & 10.013 \\
\hline FVS & 10.949 & .000 & 11.450 & 1.046 & 9.368 & 13.532 \\
\hline TMT-A & -10.517 & .000 & -66.050 & 6.280 & -78.553 & -53.547 \\
\hline TMT-B & -22.907 & .000 & -193.100 & 8.430 & -209.882 & -176.318 \\
\hline$\Sigma$ TAVR-A1/A5 & 14.486 & .000 & 25.100 & 1.733 & 21.651 & 28.549 \\
\hline TAVR-A1 & 6.165 & .000 & 2.525 & 0.410 & 1.710 & 3.340 \\
\hline TAVR-A2 & 9.203 & .000 & 3.675 & 0.399 & 2.880 & 4.470 \\
\hline TAVR-A3 & 12.581 & .000 & 5.450 & 0.433 & 4.588 & 6.312 \\
\hline TAVR-A4 & 13.384 & .000 & 6.325 & 0.473 & 5.384 & 7.266 \\
\hline TAVR-A5 & 14.389 & .000 & 7.000 & 0.486 & 6.031 & 7.969 \\
\hline TAVR-B & 9.622 & .000 & 3.475 & 0.361 & 2.756 & 4.194 \\
\hline TAVR-A6 & 13.978 & .000 & 7.450 & 0.533 & 6.389 & 8.511 \\
\hline TAVR-A7 & 14.039 & .000 & 8.075 & 0.575 & 6.930 & 9.220 \\
\hline TAVR-R & 13.272 & .000 & 7.525 & 0.567 & 6.396 & 8.654 \\
\hline
\end{tabular}


Malm Morgan, Urquijo, López, Licitra, Comesaña, Rodríguez \& Brusco I Psicodebate, 14(1) I 9-32.

\section{Tabla 6.}

Resultados de la prueba t de muestras independientes de pruebas de Toma de Decisiones en función de la pertenencia al grupo.

\begin{tabular}{|c|c|c|c|c|c|c|}
\hline \multirow{2}{*}{ Prueba } & & \multirow{2}{*}{$p$} & \multirow{2}{*}{$D M$} & \multirow{2}{*}{ EEDM } & \multicolumn{2}{|c|}{$95 \%$ IC para la diferencia } \\
\hline & & & & & Superior & Inferior \\
\hline GDT-RC* & 2.668 & .009 & 1.600 & 0.600 & 0.406 & 2.794 \\
\hline GDT-RR* $^{*}$ & -3.335 & .001 & -3.850 & 1.154 & -6.148 & -1.552 \\
\hline GDT-RS* & 3.335 & .001 & 3.850 & 1.154 & 1.552 & 6.148 \\
\hline GDT-Tpo.RS* & -1.214 & .228 & -2.200 & 1.812 & -5.808 & 1.408 \\
\hline DGT-Tpo.RR* & -1.302 & .197 & -2.250 & 1.728 & -5.690 & 1.190 \\
\hline GDT-Tpo.RP* & -2.605 & .011 & -2.700 & 1.036 & -4.763 & -0.637 \\
\hline \multicolumn{7}{|c|}{$\begin{array}{l}\text { *GDT-RC: total de respuestas correctas, GDT-RR: total de respuestas de riesgo, GDT- } \\
\text { RS: total de respuestas seguras, GDT-Tpo.RS: tiempo de reacción de respuestas segu- } \\
\text { ras, GDT-Tpo.RR: tiempo de reacción de respuestas de riesgo, GDT-Tpo.RP: tiempos de } \\
\text { reacción promedio. }\end{array}$} \\
\hline
\end{tabular}

Los resultados de los análisis estadísticos confirman que, salvo el tiempo de reacción en respuestas seguras y de riesgo en el GDT, los desempeños de los sujetos con EA son significativamente inferiores a los desempeños de los sujetos sin EA. Los resultados permiten confirmar que la toma de decisiones se ve afectada por la EA, especialmente en la cantidad de respuestas (Totales - seguras - de riesgo) y en el tiempo promedio de reacción. Con el objeto de estimar el tamaño de las diferencias observadas, se realizó un cálculo del tamaño del efecto. Los resultados se presentan en la tabla 7. 
Tabla 7.

Tamaño del efecto de las diferencias.

\begin{tabular}{|c|c|c|c|c|}
\hline Prueba & Grupo & $M$ & $D E$ & $d$ \\
\hline \multirow{2}{*}{ ADAS Cog } & C & 0.60 & 1.008 & \multirow{2}{*}{0.707} \\
\hline & $\mathrm{P}$ & 10.15 & 7.608 & \\
\hline \multirow{2}{*}{ ADAS Mem } & $\mathrm{C}$ & 4.01 & 3.175 & 0.808 \\
\hline & $\mathrm{P}$ & 11.00 & 2.991 & \\
\hline \multirow{2}{*}{ DRS } & $C$ & 138.45 & 4.188 & 0.811 \\
\hline & $\mathrm{P}$ & 115.68 & 11.155 & \\
\hline \multirow{2}{*}{ FVF } & $\mathrm{C}$ & 17.10 & 4.425 & 0.786 \\
\hline & $\mathrm{P}$ & 8.88 & 3.560 & \\
\hline \multirow{2}{*}{ FVS } & C & 21.08 & 5.413 & 0.824 \\
\hline & $P$ & 9.63 & 3.801 & \\
\hline \multirow{2}{*}{ TMT-A } & C & 39.40 & 12.087 & 0.768 \\
\hline & $P$ & 105.45 & 37.835 & \\
\hline \multirow{2}{*}{ TMT-B } & $\mathrm{C}$ & 95.75 & 31.173 & 0.932 \\
\hline & $\mathrm{P}$ & 288.85 & 43.252 & \\
\hline \multirow{2}{*}{$\Sigma$ TAVR-A1/A5 } & C & 46.48 & 7.805 & 0.872 \\
\hline & $\mathrm{P}$ & 21.38 & 7.692 & \\
\hline \multirow{2}{*}{ TAVR-A6 } & $\mathrm{C}$ & 9.63 & 2.676 & 0.868 \\
\hline & $\mathrm{P}$ & 2.18 & 2.049 & \\
\hline \multirow{2}{*}{ TAVR-A7 } & $\mathrm{C}$ & 9.85 & 2.824 & 0.894 \\
\hline & $P$ & 1.78 & 2.293 & \\
\hline \multirow{2}{*}{ TAVR-R } & $\mathrm{C}$ & 14.65 & 0.580 & 0.855 \\
\hline & $\mathrm{P}$ & 7.13 & 3.539 & \\
\hline \multirow{2}{*}{ MMSE } & $\mathrm{C}$ & 29.25 & 0.840 & 0.883 \\
\hline & $\mathrm{P}$ & 21.75 & 3.264 & \\
\hline \multirow{2}{*}{ GDT-RC* } & $\mathrm{C}$ & 8.65 & 2.833 & 0.243 \\
\hline & $P$ & 7.05 & 2.521 & \\
\hline \multirow{2}{*}{ GDT-RR* } & $\mathrm{C}$ & 7.15 & 4.441 & 0.404 \\
\hline & $\mathrm{P}$ & 11.00 & 5.796 & \\
\hline \multirow{2}{*}{ GDT-RS* } & $\mathrm{C}$ & 10.85 & 4.441 & 0.314 \\
\hline & $\mathrm{P}$ & 7.00 & 5.796 & \\
\hline \multirow{2}{*}{ GDT-Tpo.RS* } & $\mathrm{C}$ & 9.60 & 5.222 & 0.125 \\
\hline & $\mathrm{P}$ & 11.80 & 10.204 & \\
\hline \multirow{2}{*}{ DGT-Tpo.RR* } & $\mathrm{C}$ & 10.45 & 8.524 & 0.140 \\
\hline & $\mathrm{P}$ & 12.70 & 6.836 & \\
\hline \multirow{2}{*}{ GDT- Tpo.RP* } & $\mathrm{C}$ & 9.75 & 3.418 & 0.342 \\
\hline & $\mathrm{P}$ & 12.45 & 5.593 & \\
\hline
\end{tabular}


El Tamaño de Efecto (TE) es un indicador que permite hacerse una idea de cuán distintos son dos grupos en una o más variables medidas. De esta manera, cuanto mayor sea el TE, menor resulta el tamaño de la muestra que se necesita para detectar la ocurrencia poblacional de un fenómeno.

Sobre cuándo se puede considerar grande o pequeño un determinado valor suelen aceptarse las orientaciones de Cohen (1988): $d=.20$ (pequeño), $d=.50$ (moderado), $d=.80$ (grande). Estas orientaciones son un tanto arbitrarias (el autor las propone como orientación) aunque son muy aceptadas como razonables y se citan frecuentemente en la literatura científica.

Los resultados del cálculo del tamaño del efecto muestran que todas las pruebas utilizadas para evaluar deterioro cognitivo muestran un tamaño del efecto de las diferencias grande (ADAS Mem; DRS; FVS; TMT-B; $\Sigma$ TAVR-A1/A5; TAVRA6; TAVR-A7; TAVR-R; MMSE) o un tamaño del efecto de las diferencias entre moderado y grande (ADAS Cog; FVF; TMT-B).

Razonablemente, el tamaño del efecto de las diferencias para los desempeños en las pruebas de toma de decisiones se sitúa entre pequeño y moderado (GDT-RC; GDT-RR; GDT-RS; GDT-Tpo.RP) y un tamaño del efecto de las diferencias pequeño (GDT-Tpo.RS; GDT-Tpo.RR). Estos resultados confirman lo establecido anteriormente, en el sentido de que los sujetos con EA, presentan diferencias significativas, con diferencias en el tamaño de efecto más elevadas y, por ende, con mayor dificultad, en el total de respuestas de riesgo; en los tiempos de reacción promedio, en el total de respuestas seguras y en el total de respuestas correctas.

\section{Discusión}

El objeto de este estudio fue el de estudiar el patrón de deterioro del proceso de TD en personas con EA. A su vez, se intentó identificar alguna relación entre el deterioro de la TD y de las FE.

Los resultados permitieron establecer que, en los sujetos con EA incluidos en el estudio, presentaron déficits en todas las funciones cognitivas evaluadas. Cuando se comparó el desempeño de todos los tests administrados que evalúan diferentes funciones cognitivas (memoria, atención, funciones ejecutivas, praxias, gnosias entre otras), entre el grupo de sujetos del grupo control y el de sujetos con EA, el desempeño de los sujetos con EA fue significativamente inferior. Esto, una vez más, confirma lo planteado por Sinz (2008) quién sostiene que a través de la evaluación neuropsicológica se evidencia el deterioro de funciones cognitivas que no son observables en la vida diaria del sujeto que padece EA, por lo menos en sus primeros estadios. Esto sugiere que al menos en la población en estudio, tanto el proceso de TD como el resto de las FE incluidas se encuentran afectadas ya desde 
los comienzos de la EA, y esto se observa no sólo en esta investigación sino que otros estudios neuropsicológicos presentan evidencias que lo sustentan (Sitzer, Twanley, \& Jeste, 2006). Consecuentemente resulta razonable proponer que la TD y las FE en su conjunto son procesos cognitivos sensibles al deterioro cognitivo de la EA a pesar de que aún no sea posible establecer claramente el patrón de degradación paulatina de estos procesos. Al respecto, es necesario señalar que si bien las FE se encuentran afectadas desde el inicio de la EA, esta afectación estaría influyendo de alguna manera en la TD, ya sea sesgando el tipo de elección (segura/riesgo), la cantidad de respuestas o el tiempo en tomar esa decisión (Tpos. de reacción en el GDT). Esto último se confirma no sólo por los resultados observados en las investigaciones (Brand et al., 2005), sino también, teniendo en cuenta la neuropatología. En la misma se evidencia el compromiso de la CPF en general y la CPFD en particular manifestándose dificultades en la planificación, en el inicio espontáneo o deliberado de ciertas conductas, en el monitoreo y en el mantenimiento de una conducta para alcanzar una meta, la resolución de problemas y, por ende, en la TD (Coolidge, Thede, \& Jang, 2004; Rains, 2004; Choi et al., 2005; Alvarez \& Emory, 2006; Sitzer et al., 2006).

Entendiéndose que la toma de decisiones es una de las actividades más frecuentes que realizamos en la vida diaria, y que todo el tiempo estamos decidiendo; cuando una situación nos ofrece más de una opción, se manifiesta un conflicto en función de las diferentes posibles consecuencias resultado de la decisión a tomar. Por ello, este estudio focalizó la atención en la TD bajo riesgo, ya que pareciera posible que la existencia de reglas explícitas ayudarían de alguna manera, como una guía para un mejor funcionamiento de las FE y, por lo tanto, podría mejorar la TD en sujetos que padecen la EA (Brand, et. al., 2007; Brand, et. al., 2008).

Por lo tanto, los resultados de esta investigación aportan evidencia empírica y dan peso a los supuestos planteados por Brand (2005), confirmando que la TD estaría relacionada directamente con las FE. Esto a su vez se relacionaría con lo que Brand (2008) denomina como estrategia cognitiva, donde están involucrados los componentes cognitivos necesarios como las FE para poder decidir de manera ventajosa. Entonces, si esta estrategia cognitiva se encuentra afectada y por lo tanto las FE también lo están desde el comienzo en la EA, es posible deducir que la TD también muestra un compromiso gradual de deterioro desde el inicio de la enfermedad.

Los resultados de esta investigación aportan evidencias empíricas a favor de esta última hipótesis, cuando ya se diagnostica la EA en un estadio de deterioro leve. La interpretación de los datos sugiere que no sólo se ve implicada la elección del tipo de respuesta (segura o de riesgo), con una mayor tendencia a tomar decisiones más riesgosas que las personas sanas, sino también, el tiempo de reacción frente a la 
respuesta (con un aumento significativo en pacientes con EA). Estos datos permiten sustentar que la TD en situaciones de riesgo se ve afectada por la EA y que la misma estaría directamente vinculada con otras FE (Brand, et al., 2007).

En la vida cotidiana, una persona que cursa la EA en los primeros estadios podría tomar una decisión simple con ayuda de una guía y respetando el tiempo que necesite para tomar esa decisión, pero cuando presenta un grado de deterioro leve de la enfermedad, la capacidad para tomar decisiones trascendentes de su vida comienza a verse afectada. Esto nos lleva a considerar el valor clínico potencial de la evaluación de la capacidad de TD ya desde la sospecha de los primeros síntomas compatibles con la EA. La literatura científica cita evidencias de que quien padece una EA no puede generar estrategias satisfactorias ante la TD (Delazer et al., 2007; Sinz et al., 2008). Los resultados obtenidos en esta investigación aportan evidencias empíricas que sugieren que no sólo parece observarse una tendencia a elegir mayoritariamente respuestas con consecuencias riesgosas, sino también, que el tiempo para tomar esa decisión parece aumentar de manera significativa. Esto último podría relacionarse con la capacidad de mantener la atención y de realizar el monitoreo de la situación a decidir, componentes relacionados con las FE, y sabiendo que éstas están afectadas desde los comienzos de la enfermedad interfieren en la TD.

Una de las características de la EA observada en este estudio es que las personas afectadas no demuestran capacidad de evaluación ni reflexión sobre las circunstancias como para tomar una decisión adecuada. Por lo tanto, es importante destacar el hecho de que existen evidencias sobre las características de la EA que tienen implicaciones de relevancia en el campo legal, sobre todo, para determinar cuándo sería el límite para decidir o establecer que una persona afectada por la EA pueda o no tomar decisiones trascendentales sobre su vida y/o sus bienes. Si de acuerdo a los resultados obtenidos en esta investigación se infiere que quien padece de EA tendría una tendencia a tomar decisiones con mayor riesgo que podrían llegar a afectar su patrimonio, y las mismas devienen de una incapacidad para evaluar con detalle cuales serían las consecuencias de sus decisiones, sería prudente destacar la importancia de realizar estudios más amplios y detallados para determinar los efectos de la enfermedad sobre la TD.

Si bien este es un estudio acotado sobre la TD y la EA, se considera que brinda un aporte a los profesionales de la salud muy interesante a la hora de plantear a familiares de un sujeto que padece la EA las limitaciones que se imponen a medida que la enfermedad avanza en cuanto a la capacidad de dicho sujeto de tomar decisiones y tener en cuenta también, en desarrollar estrategias de estimulación cognitiva que permitan mantener la capacidad de TD el mayor tiempo posible durante el transcurso de la EA. 


\section{Referencias}

Allegri, R. F., Ollari, J. A., Mangone, C.A., Arizaga, R. L., De Pascale, A., Pellegrini, M., ... Taragano, F. E. (1999). El "Mini-Mental State Examination" en la Argentina: Instrucciones para su administración. Grupo de Trabajo de Neuropsicología Clínica de la Sociedad Neurológica Argentina. Revista Neurológica Argentina, 24, (1), 31-35.

Alvarez, J. \& Emory, E. (2006). Executive Function and the Frontal Lobes: A Meta Analytic review. Neuropsychology Review, 16(1), 17-42.

Barkley, R. A. (2001). The Executive Functions and Self-regulation: An Evolutionary Neuropsychological Perspective. Neuropsychology Review, 11(1), 1-29.

Bechara, A., Damasio, A. R., Damasio, H., \& Anderson, S. W. (1994). Insensitivity to future consequences following damage to human prefrontal cortex. Cognition, 50(1-3), 7-15.

Bechara, A., Damasio, H., Tranel, D., \& Damasio, A. R. (2005). The Iowa Gambling Task and the somatic marker hypothesis: some questions and answers. Trends in Cognitive Sciences, 9(4), 159-162.

Bechara, A. (2006). Neural Basis of Decision-Making and Implications for Older Adults. The Center for Decision Neuroscience Institute for the Neurological Study of Emotion, Decision-Making, and Creativity University of Southern California. The National Academic Press.

Bermejo-Pareja, F., Benito-Leon, J., Vega, S., Medrano, M. J., \& Roman, G. C. (2008). Incidence and subtypes of dementia in three elderly populations of central spain. Journal of the Neurological Sciences, 15, 264, (1-2), 63-72.

Bonatti, E., Zamarian, L., Wagner, M., Benke, T., Hollosi, P., Strubreither, W., \& Delazer, M. (2008). Making Decisions and Avising Decisions in Trumatic Brain Injury. Cognitive Behavioural Neurology, 21, 164-175.

Bonatti, E., Kuchukhidze, G., Zamarian, L., Trinka, E., Bodner, T., Benke, T., \& Delazer, M. (2009). Decision making in ambiguous and risky situations after unilateral temporal lobe epilepsy surgery. Epilepsy \& Behavior, 14, 665-673.

Brand, M. (2008). Does the feedback from previous trials influence current decisions? A study on the role of feedback processing in making decisions under explicit risk conditions. Journal of Neuropsychology, 2, 431-443. 
Brand, M., Fujiwara, E., Borsutzky, S., Kalbe, E., Kessler, J., \& Markowitsch, H. J. (2005). Decision-Making Deficits of Korsakoff Patients in a New Gambling Task with Explict Rules: Associations with Executive Functions. Neuropsychology, 19(3), 267-277.

Brand, M., Grabenhorst, F., Starcke, K., Vandekerckhove, M. M. P., \& Markowitsch, H. J. (2007). Role of the amygdale in decisions under ambiguity and decisions under risk: Evidence from patients with Urbach-Wiethe disease. Neuropsychologia, 45, 1305-1317.

Brand, M., Heinze, K., Labudda, K., \& Markowitsch, H. J. (2008). The role of strategies in deciding advantageously in ambiguous and risky situations. Cognitive Process, 9, 159-173.

Brand, M., Kalbe, E., Labudda, K., Fujiwara, E., Kessler, J., \& Markowitsch, H. J. (2005). Decision-making impairments in patients with pathological gambling. Psychiatry Research, 133, 91-99.

Brand, M., Labudda, K., Kalbe, E., Hilker, R., Emmans, D., Fuchs, G., Kessler, J., \& Markowitsch, H. J. (2004). Decision-making impairments in patients with Parkinson's disease. Behavioural Neurology, 15, 77-85.

Brand, M., Labudda, K., \& Markowitsch, H. J. (2006). Neuropsychological correlates of decision-making in ambiguous and risky situations. Neural Networks, 19, 1266-1276.

Brand, M., Laier, C., Pawlikowski, M., \& Markowitsch, H. J. (2009). Decision making with and without feedback: the role of intelligence, strategies, executive functions, and cognitive styles. Journal of Clinical and Experimental Neuropsychology, 31(8), 984-998.

Brand, M., Recknor, E. C., Grabenhorst, F., \& Bechara, A. (2006). Decisions under ambiguity and decisions under risk: correlations with executive functions and comparisons of two different gambling tasks with implicit and explicit rules. Journal of Clinical and Experimental Neuropsychology, 29(1), 86-99.

Brand, M., Recknor, E. C., Grabenhorst, F., \& Bechara, A. (2007). Decisions under ambiguity and decisions under risk: correlations with executive functions and comparisons of two different gambling tasks with implicit and explicit rules. Journal of Clinical and Experimental Neuropsychology, 29(1), 86-99. 
Brand, M., Pawlikowski, M., Labudda, K., Laier, C., von Rothkirch, N., \& Markowitsch, H. J. (2009). Do amnesic patients with Korsakoff's syndrome use feedback when making decisions under risky conditions? An experimental investigation with the Game of Dice Task with and without feedback. Brain and Cognition, 69, 279-290.

Bufill, E., Bartés, A., Moral, A., Casadevall, T., Codinachs, M., Zapater, E., Carles Rovira, J., Roura, P., Oliva, R., \& Blesa, R. (2009). Factores genéticos $\mathrm{y}$ ambientales que pueden influir en la forma senil de la enfermedad de Alzheimer: estudio de casos y controles anidado. Neurología, 24(2), 108-112.

Burin, D., Drake, M., \& Harris, P. (2007). Evaluación Neuropsicológica en Adultos. Bs. As., Argentina: Paidós.

Choi, S. J.; Lim, K. O.; Monteiro, I. y Reisberg, B. (2005). Diffusion Tensor Imaging of Frontal White Matter Microstructure in early Alzheimer's disease: A Preliminary Study. Journal of Geriatric Psychiatry and Neurology, 18, 12-19.

Clark, L., Manes, F., Antoun, N., Sahakian, S. J., \& Robbins, T. (2003). The contributions of lesions laterality and lesion volume to decision-making impairment following frontal lobe damage. Neuropsychologia, 41, 1474-1483.

Cohen, J. (1988). Statistical Power Analysis for the Behavioral Sciences (2da ed.). Erlbaum: Hillsdale, N.J.

Coolidge, F., Thede, L., \& Jang, K. (2004). Are Personality disorders psychological Manifestations of Executive Function deficits? Bivariate Heritability Evidence From twin Study. Behavior Genetics, 34(1), 75-84.

Delazer, M., Sinz, H., Zamarian, L., \& Benke, T., (2007). Decision-making with explicit and stable rules in mild Alzheimer's disease. Neuropsychologia, 45(8), 1632-1641.

Delazer, M., Sinz, H., Zamarian, L., Stockner, H., Seppi, K., Wenning, G. K., Benke, T., \& Poewe, W. (2009). Decision making under risk and under ambiguity in Parkinson's disease. Neuropsychologia, 47, 1901-1908.

Delazer, M., Zamarian, L., Bonatti, E., Kuchukhidze, G., Koppelstatter, F., Bodner, T. \& Trinka, E. (2010). Decision-making under ambiguity and under risk inmesial temporal lobe epilepsy. Neuropsychologia, 48, 194-200. 
Di Carlo, A., Baldereschi, M., Amaducci, L., Lepore, V., Bracco, L., Maggi, S., ... Inzitari, D. (2002). Incidence of dementia, Alzheimer's disease and vascular dementia in Italy. The ILSA study. Journal of the American Geriatrics Society, 50(1), 41-48.

Duke, L. M. \& Kaszniak, A. W. (2000). Executive Control Functions in Degenerative Dementias: A Comparative Review. Neuropsychology Review, 10(2), 75-99.

Euteneuer, F., Schaefer, F., Stuermer, R., Boucsein, W., Timmermann, L., Barbe, M., ... Kalbe, E. (2009). Dissociation of decision-making under ambiguity and decisionmaking under risk in patients with Parkinson's disease: A neuropsychological and psychophysiological study. Neuropsychologia, 47, 2882-2890.

Formiga, F., Fort, I., Robles, M. J., Riu, S., Rodríguez, D. \& Sabartes, O. (2008). Aspectos diferenciales de comorbilidad en pacientes ancianos con demencia tipo Alzheimer o con demencia vascular. Revista de Neurología, 46(2), 72-76.

Labudda, K., Woermann, F. G., Mertens, M., Pohlmann-Eden, B., Markowitsch, H. J., \& Brand, M. (2008). Neural correlates of decisions making with explicit information about probabilities and incentives in elderly healthy subjects. Exp Brain Res, 187, 641-650.

Lezak, M. (1995) Neuropsychological Assessment (3ra ed.). New York: Oxford University Press.

Manes, F., Sahakian, B., Clark, L., Rogers, R., Antoun, N., Aitken, M. \& Robbins, T. (2002). Decision-making processes following damage to de prefrontal cortex. Brain, 125, 624-639.

Mangone, C. A. \& Sanguinetti, R. (1997). Enfermedad de Alzheimer. En C. A. Mangone, R. F. Allegri, R. L. Arizaga, \& J. A. Ollari (Eds.) Demencia. Enfoque multidisciplinario, 119-142. Buenos Aires, Argentina: Sagitario.

Martín-Carrasco, M. (2009). Biomarcadores en la Enfermedad de Alzheimer: definición, significación diagnóstica y utilidad clínica. Psicogeriatría, 1(2), $101-114$.

Muñoz-Céspedes, J. M. \& Tirapu-Ustárroz, J. (2004). Rehabilitación de las funciones ejecutivas. Revista Neurológica, 38(7), 656-663.

Perry, R. J. \& Hodges, J. R. (1999). Attention and executive deficits in Alzheimer's disease. A critical review. Brain, 122, 383-404. 
Pineda, D. A. (2000). La función ejecutiva y sus trastornos. Revista Neurológica, 30(8), 764-768.

Rains, G. D. (2004). Principios de Neuropsicología Humana. México: Mc Graw Hill.

Rogers, R. D., Owen, A. M., Middleton, H. C., Williams, J., Pickard, J. D., Sahakian, B. J., \& Robbins, T. W. (1999). Choosing between small, likely rewards and large, unlikely rewards activates inferior and orbital prefrontal cortex. The Journal of Neuroscience, 20(19), 9029-9038.

Rossi, M., Gerschcovich, E. R., de Achaval, D., Perez-Lloret, S., Cerquetti, D., Cammarota, A., Nouzeilles, M. I., Fharer, R., Merello, M., \& Leiguarda, R. (2010). Decision-making in Parkinson's disease patients with and without pathological gambling. European Journal of Neurology, 17, 97-102.

Sastre-Riva, S. (2006). Condiciones tempranas del desarrollo y el aprendizaje: el papel de las funciones ejecutivas. Revista de Neurología, 42(2), 143-151.

Semenova, O. A. (2005). Problems of Studying Executive Functions of Mental Activity in Humans. Human Physiology, 31(6), 715-713.

Shannon Rogan, B. S. \& Lippa, C. F. (2002). Alzheimer's disease and others dementias: a review. American Journal of Alzheimer's Disease and other Dementias, 17(1), 11-17.

Sinz, H., Zamarian, L., Benke, T., Wenning, G. K. \& Delazer, M. (2008). Impact of ambiguity and risk on decision making in mild Alzheimer's disease. Neuropsychologia, 46, 2043-2055.

Sitzer, D. I., Twamley, E. W. \& Jeste, D. V. (2006). Cognitive training in Alzheimer's disease: a meta-analysis of the literature. Acta Psychiatr Scand, 114, 75-90.

Tirapu-Ustárroz, J., Muñoz-Céspedes, J. M., Pelegrín-Valero, C., \& AlbénizFerreras, A. (2005). Propuesta de un protocolo para la evaluación de las funciones ejecutivas. Revista Neurológica, 41(3), 177-186. 
